\title{
Penetrating Behavior of a Single Particle Injected into Liquid $^{*}$
}

\section{By Jueng-Gil LEE** and Masanori TOKUDA***}

\begin{abstract}
Synopsis
The penetrating behavior of a single particle impinged on liquid surface has been studied with cold model experiments.

The velocity of a polystyrene particle at impact on distilled water or glycerin liquid was obtained by detectors consisted of laser diode-photo sensors. The behavior of the particle, entrained gas bubbles and liquid flow were observed by using a high speed video and camera and analyzed by a mathematical model.

At impact of the particle on liquid surface, a cavity was formed and gas was entrained as a columnar bubble, when the particle impact velocity $\left(V_{p o}\right)$ exceeded a critical velocity.

Although the penetrating length of the particle $\left(L_{p}\right)$ increased with increasing $V_{p o}$, the rate of increase of $L_{p}$ gradually decreases with increasing $V_{p o}$.

A mathematical model was developed to predict how the initial kinetic energy of the particle was distributed and consumed by several energy dissipation mechanisms.

It was inferred that most of the kinetic energy was consumed as dissipation works, mainly spitting and heat generation in a higher region of $V_{p o}$ and hence the attainment of a higher particle velocity could not be an effective means to increase penetration length into the liquid.

The effect of reduction of the apparent liquid density by increasing gas holdup in the liquid with entrained gas or gas jet was discussed as an effective way to obtain deep penetration of powders into the liquid.
\end{abstract}

Key words: powder injection; high speed video; penetration behavior; particle; impact velocity; columnar bubble; penetration depth; entrained gas; gas holdup; mathematical model; cavity; energy dissipation.

\section{Introduction}

The injection of powder into melts, injection metallurgy, has become increasingly important to metallurgical refining processes.

Although the injection process is now being used successfully and clearly has considerable potential, very little work has been reported on the penetrating behavior of powders in melts.

In the last few years, the research papers on the penetrating behavior of a single particle or powder injection can be divided into the following four subjects ;

1) penetration length ${ }^{1,2)}$

2) critical condition for penetration of solid particles $^{3,4)}$

3) trajectory of gas/particulate solids jet in a melt ${ }^{5)}$

4) bubbling/jetting transition for gas-particle injection. ${ }^{2,6-8)}$

The researches outlined above have following limitations.

(1) When particulate solids were injected, parti- cle velocity at the liquid surface just before penetration was not measured.

(2) Although Ozawa and Mori ${ }^{4)}$ calculated the critical conditions for sphere penetration into liquid metal by considering cavity formation according to curve fitting, the penetration behavior of a high speed particle above these critical conditions was not clarified.

(3) In the correlation between single particle and powder injection no report has discussed the entrainment of gas that occurs with powder injection.

(4) Although some works have been reported on penetration depth as a characteristic of penetration behavior with powder injection, a penetration mechanism closely correlated with reaction efficiency, that is whether the penetrating particle passed through the gas-liquid interface or became trapped inside a gas bubble in the liquid, has not been clarified and there has been no report on the residence times of penetrating particles in liquids.

For the reasons outlined above, we have attempted to clarify penetration behavior of a high speed particle with measurement of the penetration length by using a high speed video system and particle velocity by using a specially designed detector.

On the basis of the analysis of the behavior of single particles, penetration behavior in powder injection has been also discussed.

\section{Experiment}

The experimental apparatus consisted of a particle accelerator, a velocity measurement and an observation system. A schematic drawing is shown in Fig. 1. The inner dimension of the glass vessel was 15 $\mathrm{cm}$ width, $12 \mathrm{~cm}$ length and $33 \mathrm{~cm}$ height.

Experiments were performed in the distilled waterpolystyrene system (dia. $3,2 \mathrm{~mm}$ ) and the glycerinpolystyrene system $(3,2 \mathrm{~mm})$. Table 1 lists the physical properties of the solid particle and the liquid.

The particle accelerating system consisted of a pressurized nitrogen reservoir, a magnetic valve and a vacuum loader. The particle was loaded through the nozzle by the vacuum. After the pressurized nitrogen was introduced into the gas reservoir up to a prescribed pressure, the loaded particle was discharged from the nozzle by instantaneous opening of a magnetic valve. The nozzle diameters were 3.1 and 2.1

* Based on the papers presented to the 112th ISIJ Meeting, October 1986, S955, at Nagoya University in Nagoya and to the 114th ISIJ Meeting, October 1987, S945, at Kumamoto Institute of Technology in Kumamoto. Manuscript received on September 9, 1987; accepted in the final form on December 11, 1987.

** Graduate Student, Faculty of Engineering, Tohoku University, Aza-Aoba, Aramaki, Sendai 980.

*** Research Institute of Mineral Dressing and Metallurgy, Tohoku University, Katahira, Sendai 980. 


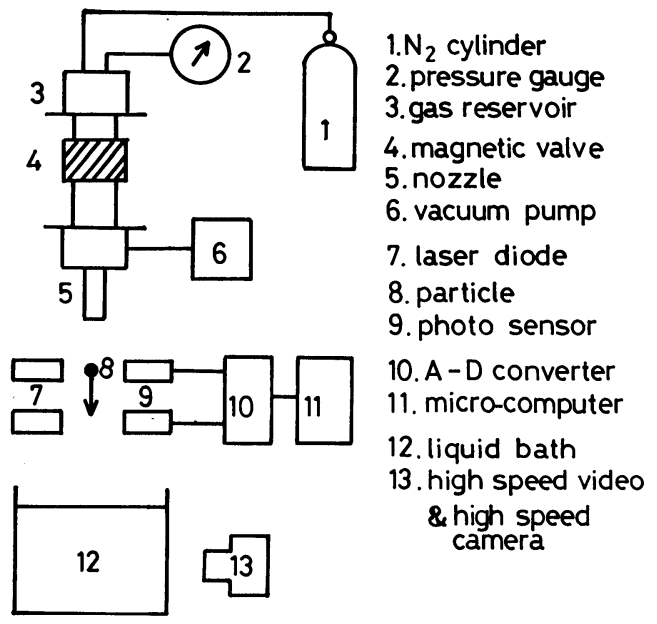

Fig. 1. Schematic drawing of the experimental apparatus.

Table 1. Physical properties of solid particle and liquids.

\begin{tabular}{llcc}
\hline & $\begin{array}{l}\text { Density } \\
\left(\mathrm{g} / \mathrm{cm}^{3}\right)\end{array}$ & $\begin{array}{c}\text { Viscosity } \\
(\mathrm{cp})\end{array}$ & $\begin{array}{c}\text { Surface } \\
\text { tension } \\
(\mathrm{dyn} / \mathrm{cm})\end{array}$ \\
\hline Polystyrene & 0.974 & & \\
Distilled water $\left(20^{\circ} \mathrm{C}\right)$ & 0.99823 & 1.002 & 72.75 \\
Glycerin $\left(30^{\circ} \mathrm{C}\right)$ & 1.260 & 629 & 61.8 \\
Glycerin $\left(20^{\circ} \mathrm{C}\right)$ & 1.260 & 1490 & 63.4 \\
\hline
\end{tabular}

Table 2. Relation between current and gas holdup.

\begin{tabular}{ccccc}
\hline Current $(\mathrm{mA})$ & 50 & 100 & 150 & 200 \\
\hline Gas holdup $(-)\left(\times 10^{-4}\right)$ & 1.6 & 3.2 & 4.8 & 6.4 \\
\hline
\end{tabular}

$\mathrm{mm}$ corresponding to the particle diameters 3 and 2 $\mathrm{mm}$, respectively.

The detector for velocity measurement consisted of two pairs of laser diode-photo sensors, which were separated from each other by a distance of $62.4 \mathrm{~mm}$.

The velocity of an accelerated particle was determined by measuring its traveling time through the two detecting points. The velocity of the particle at the liquid surface, the impact velocity, was calculated by the force balance in air. It was confirmed by measurement of the velocity with the lower detector at the level of liquid surface.

A high speed video (200 frames/s) was employed to observe the penetration behavior and measure the penetration lengths of the particle and residence time in liquid.

A high speed camera (2 000 frames/s) was also employed to get an image of interaction between the penetrating particle and its surrounding.

The effect of gas holdup in the liquid on particle penetration length was also examined.

The apparatus was identical to that used for the single particle impinging system except that an electrolysis system with Pt electrodes was installed which enabled to supply a uniform and steady state gas holdup in $0.01 \% \mathrm{KCl}$ solution. Table 2 gives the relationship between the electric current of the circuit and corresponding values of gas holdup $H(-)$. The gas holdup was determined from the measurement of the volume of gas containing liquid introduced in a burette which was hold in the vessel.

\section{Results}

\section{Observation of Penetration Behavior}

Figure 2 represents penetration behavior with the lapse of time for the polystyrene (dia. $3 \mathrm{~mm}$ )-distilled water system. In this case, the particle velocity at the liquid surface just before penetration into the liquid was $25 \mathrm{~m} / \mathrm{s}$.

Figure 2(a) shows the phenomena right after penetration of a polystyrene particle in distilled water. Cavity formation occurred and a cylindrical wave was formed at the periphery of the cavity. The particle was located in the center of the cavity.

If the particle velocity was lower than a critical velocity $(13 \sim 14 \mathrm{~m} / \mathrm{s}$, for the $3 \mathrm{~mm}$ polystyrene-water system), only the particle penetrated slowly into the interior of the liquid, while the cavity collapsed.

However, if the particle impact velocity was greater than the critical velocity, the behavior of particle penetration follows the photographs as shown in Figs. 2(b) to 2(f).

Figures 2(b) and 2(c) show the penetration of the particle and the formation of a columnar bubble with a lapse of 5 and $10 \mathrm{~ms}$ after particle impingement on the liquid surface, respectively.

The cavity elongated to become a columnar bubble, which moved into the distilled water. The particle, as shown in Figs. 2(c) to 2(e) was separated from the columnar bubble. This separation occurred almost always at about $30 \mathrm{~mm}$ from the original liquid surface (The lines in photos indicate the distance from the surface in $\mathrm{cm}$ ).

After this separation, the particle moved slowly into the liquid, and the columnar bubble was broken into some parts and the tip of the bubble penetrated more deeply into the liquid as shown in Figs. 2(d) and 2(e).

These photos also clearly show floating up of the upper parts torn off from the main columnar bubble due to the loss of driving force for penetration. The columnar bubble disintegrated into small bubbles and dispersed into the liquid. The total residence time for the bubbles from cavity formation to return to the free surface was $345 \mathrm{~ms}$.

The particle, on the other hand, still kept penetrating into the liquid. Although the penetration length of the particle was shorter than the bubble, the residence time of the particle was much larger. It was approximately $2.3 \mathrm{~s}$ according to the measurement by the high speed camera for this particular case.

Figure 3 shows penetration behavior in glycerinpolystyrene system at $30^{\circ} \mathrm{G}$.

The size of a cavity which accompanied penetration of a particle was approximately $2 \sim 5$ times larger than the particle diameter itself and it increased with increasing particle velocity. 

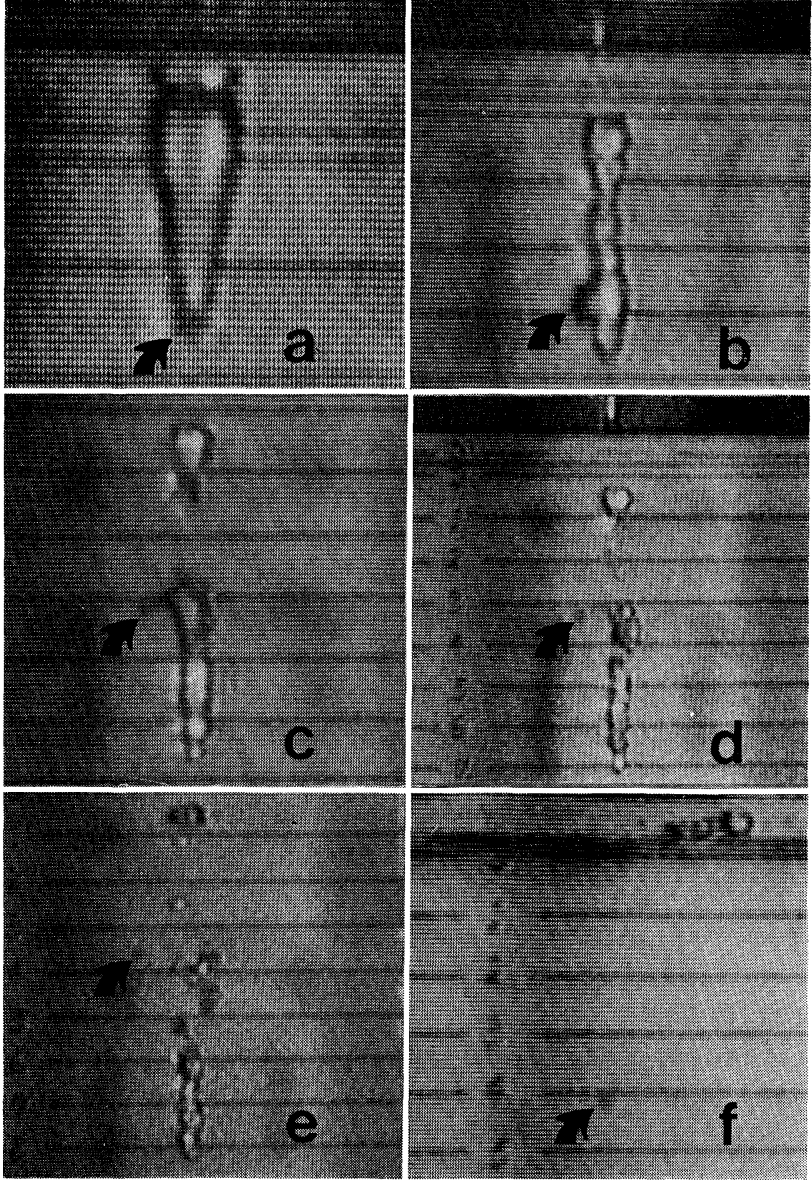

The line interval in each figure corresponds to $10 \mathrm{~mm}$.

$V_{p o}=25 \mathrm{~m} / \mathrm{s}$
Elapsed time:
(a) $0 \mathrm{~ms}$
(b) $5 \mathrm{~ms}$
(c) $10 \mathrm{~ms}$
(d) $15 \mathrm{~ms}$
(e) $25 \mathrm{~ms}$
(f) $345 \mathrm{~ms}$

The arrow indicates the particle.

Fig. 2. Observations of the penetrating behavior in polystyrene-distilled water system in different magnification.

The penetration length was shorter and residence time of a particle $(6 \mathrm{~s})$ and gas $(2.4 \mathrm{~s})$ were longer because of the higher viscosity as compared with distilled water. The penetration behavior was similar to that of the distilled water.

To clarify the entrainment mechanism of the columnar bubble and the liquid flow around columnar bubble, red ink was employed as a tracer of moving liquid. A $1.5 \mathrm{~cm}$ layer of red ink was placed on the top of glycerin and flow of red ink was traced by a high speed camera.

Figure 4(a) shows that a columnar black part is formed by penetration of the particle. This is assumed to correspond to the columnar bubble in Figs. 2(c) and 3(c).

The columnar bubble is surrounded by something different from the bulk glycerin liquid. Figure 4(d) represents the state after the lapse of $7 \mathrm{~s}$, when all of the entrained gas disappear by floating up and only a thin layer of ink remains in the bulk of glycerin.

It means that the deeply penetrating columnar bubble is surrounded by the ink from free surface and

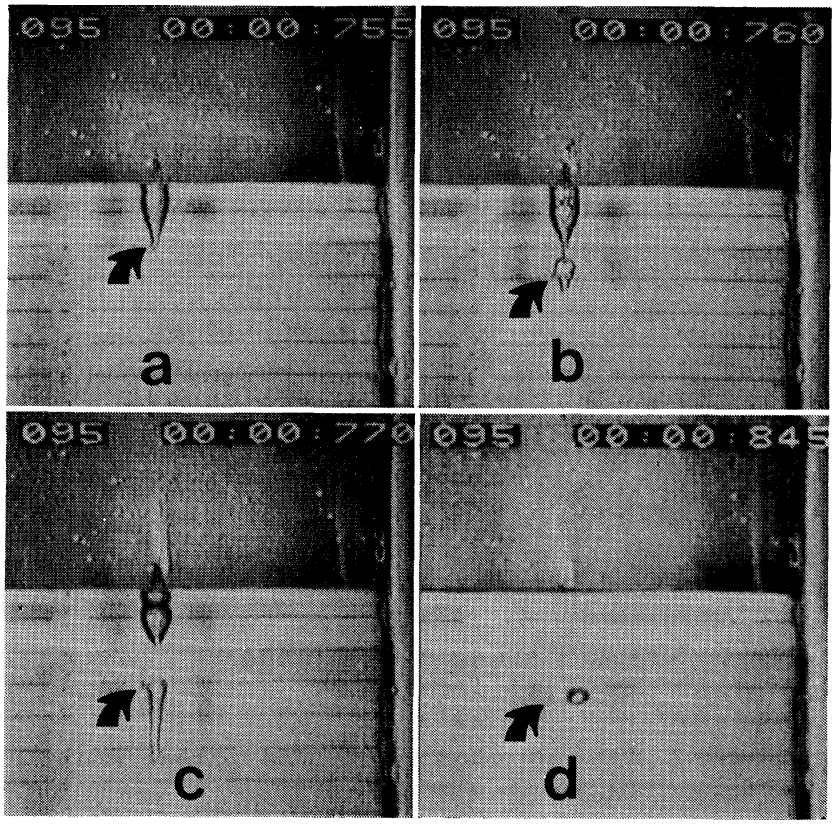

$V_{p o}=28 \mathrm{~m} / \mathrm{s}$

Elapsed time: (a) $0 \mathrm{~ms}$ (b) $5 \mathrm{~ms}$ (c) $15 \mathrm{~ms}$

(d) $90 \mathrm{~ms}$

The arrow indicates the particle.

Fig. 3. Observations of the penetrating behavior in polystyrene-glycerin system.

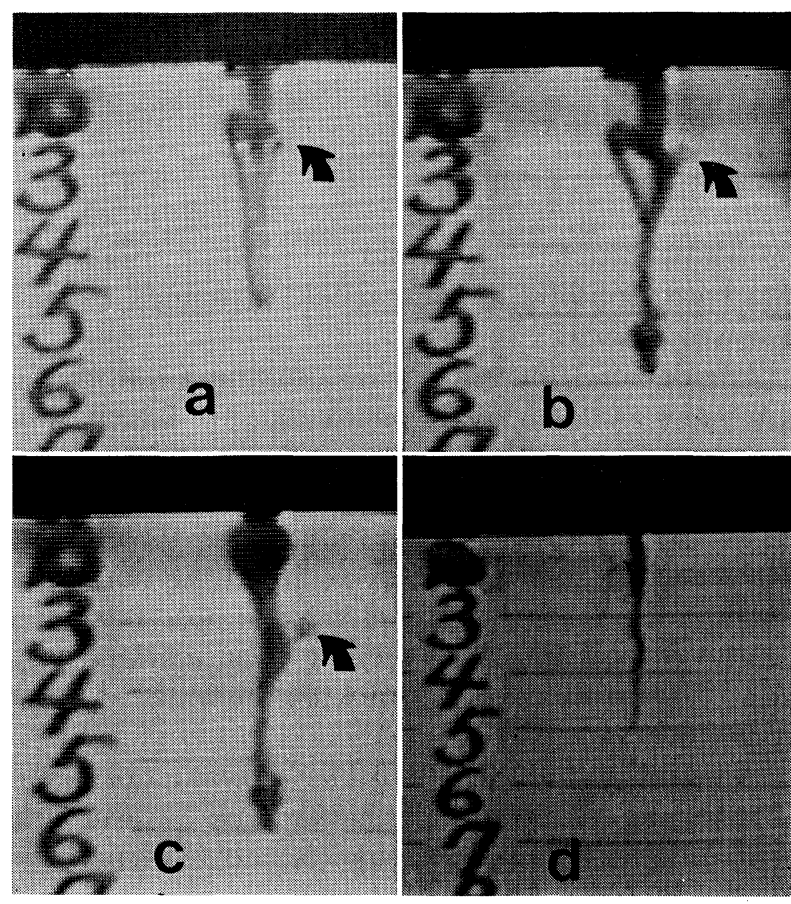

$V_{p o}=35 \mathrm{~m} / \mathrm{s}$

Elapsed time: (a) $15 \mathrm{~ms}$ (b) $30 \mathrm{~ms}$ (c) $50 \mathrm{~ms}$ (d) $7 \mathrm{~s}$

The arrow indicates the particle.

Fig. 4. Observation of the penetrating phenomena of the columnar bubble accompanied with thin liquid boundary layer moving into the liquid.

the layer of ink simultaneously moves into the liquid in company with penetration of the columnar bubble. 


\section{The Effect of Particle Velocity on Cavity Formation}

For velocities less than a critical impact velocity only cavity formation took place and penetration of a columnar bubble did not occur. Figure 5 shows that cavities with a diameter $2 \sim 3$ times larger than that of the particle did form and their size were not significantly influenced by impact velocity.

\section{The Effect of Particle Velocity on Gas Holdup}

The gas holdup, i.e., the gas volume entrained with particle penetration as a columnar bubble, was obtained by image analyses of the photos from a high speed video. The relation between gas holdup and particle velocity at the liquid surface $\left(V_{p o}\right)$ is shown in Fig. 6. Although there is a wide scattering especially in the smaller region of $V_{p o}$, it is clear that the large volume of gas entrained into liquid and the total volume reached to $50 \sim 70$ times larger than the volume of the particle itself in the higher region of $V_{p o}$.

\section{Dependence of Penetration Length on Particle Velocity}

\section{Distilled Water-Polystyrene System}

The effects of particle impact velocity on the penetration lengths of 3 and $2 \mathrm{~mm}$ polystyrene particles (-) and gas (O) are shown in Figs. 7 and 8, respectively.

As shown in Fig. 7, a columnar bubble with 1.5

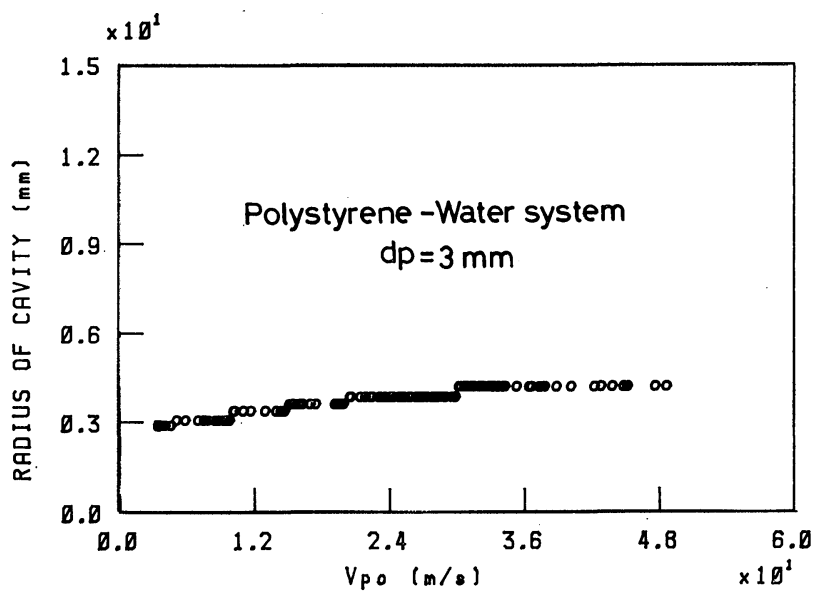

Fig. 5. Relation between the cavity radius and impact velocity $\left(V_{p o}\right)$

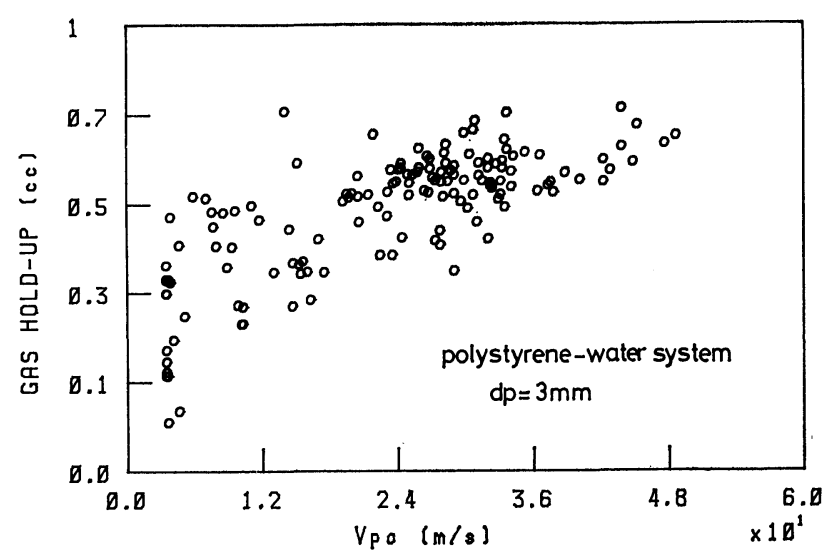

Fig. 6. Variation of gas holdup with impact velocity $\left(V_{p o}\right)$. times larger diameter than the particle penetrated more deeply into liquid than the particle, when the particle velocity at the liquid surface was larger than $13 \sim 14 \mathrm{~m} / \mathrm{s}$. The penetration length of the particle did not increase substantially with increasing particle velocity (Fig. 7).

In case of the smaller particle $(2 \mathrm{~mm})$, as shown in Fig. 8, the critical velocity of the particle beyond which the bubble penetrates deeper than the particle is around $16 \sim 17 \mathrm{~m} / \mathrm{s}$. The penetration length of the particle $(2 \mathrm{~mm})$ was smaller than that of the $3 \mathrm{~mm}$ particle as a result of the decrease in momentum with decreasing particle size.

The dashed curves in Figs. 7 to 10 represent the hypothetical penetration length of a particle calculated by a force balance as is discussed in Sec. IV. 1 . The experimental penetration depth of a particle was approximately 0.6 times the hypothetical penetration length. The difference between the experimental and hypothetical value increased with increasing particle velocity.

\section{Glycerin-Polystyrene System}

The effect of impact velocity on penetration length of 3 and $2 \mathrm{~mm}$ polystyrene particles (0) and gas bubbles $(\bigcirc)$ is shown in Figs. 9 and 10, respectively.

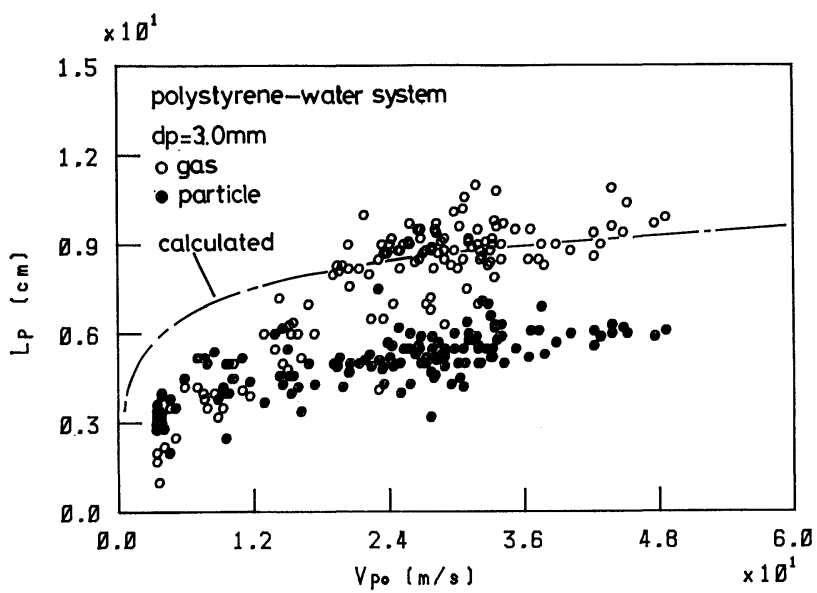

Fig. 7. Change of the penetration lengths of gas and particle $\left(L_{p}\right)$ with impact velocity $\left(V_{p o}\right)$.

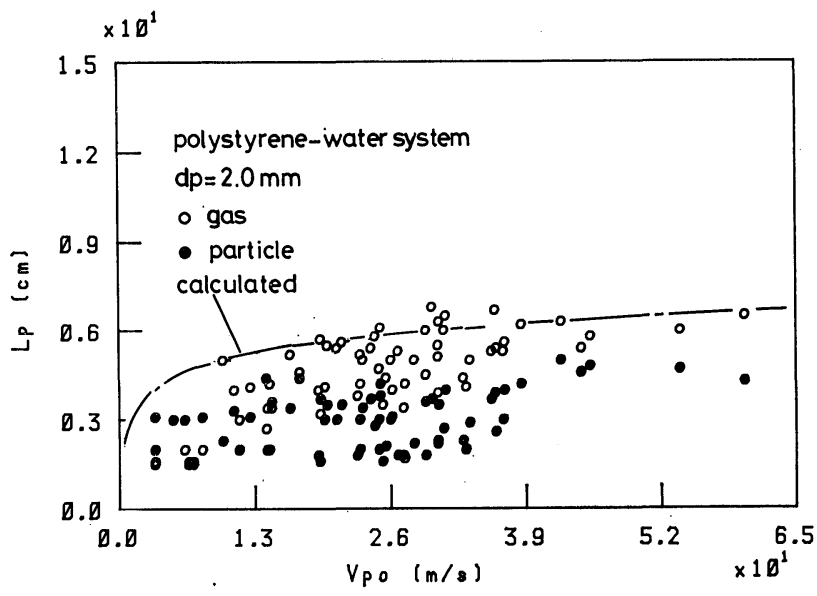

Fig. 8. Change of the penetration leng ths of gas and particle $\left(L_{p}\right)$ with impact velocity $\left(V_{p o}\right)$. 


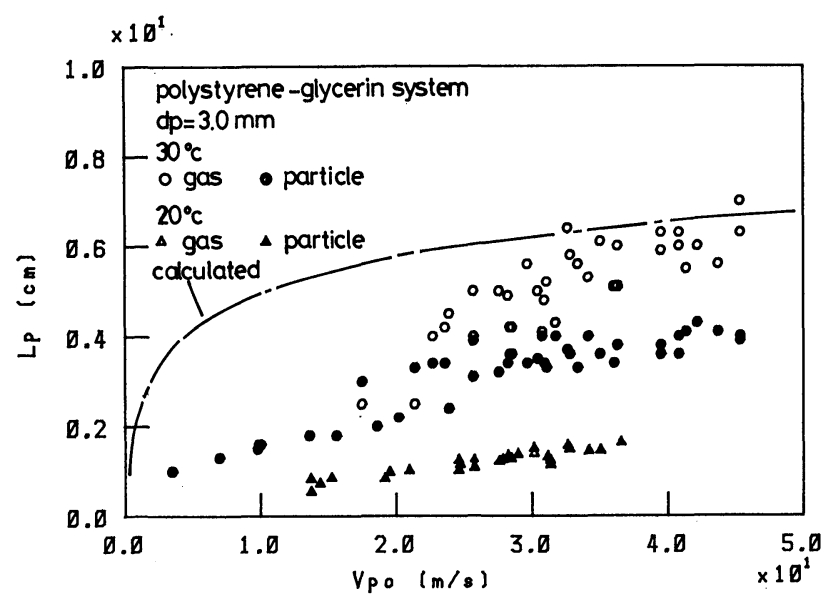

Fig. 9. Change of the penetration lengths of gas and particle $\left(L_{p}\right)$ with impact velocity $\left(V_{p o}\right)$.

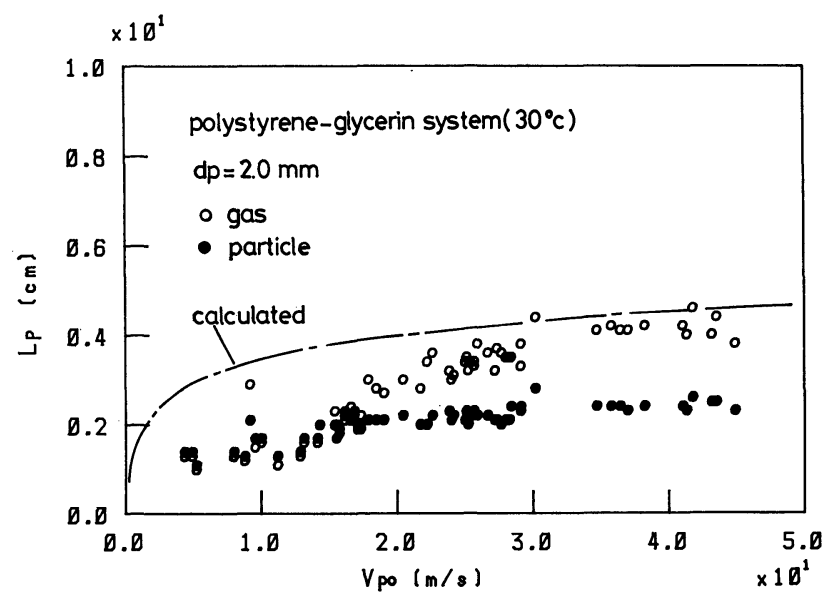

Fig. 10. Change of the penetration lengths of gas and particle $\left(L_{p}\right)$ with impact velocity $\left(V_{p o}\right)$.

Figure 9 shows results of experiment at 20 and $30^{\circ} \mathrm{C}$.

At $30^{\circ} \mathrm{C}$, the critical particle velocity for separation of the gas bubble and particle was $21 \mathrm{~m} / \mathrm{s}$. Although there is a marked difference in the viscosity between glycerin and distilled water as shown in Table 1, penetration behavior seems to be similar in both systems.

The diameter of the columnar bubble was approximately 1.2 times of particle diameter and the experimental penetration length was $0.5 \sim 0.6$ times of the hypothetical value.

At $20^{\circ} \mathrm{C}$, the penetration behavior of the columnar bubble was not observed. This was because the viscosity of glycerin at $20^{\circ} \mathrm{C}$ is 2.37 times bigger than that of $30^{\circ} \mathrm{C}$.

The effect of the size of the particle (2 and $3 \mathrm{~mm}$ ) on the penetration behavior was not significant.

The penetration behavior was also examined for a particle with greater density than water. When an alumina particle of $3 \mathrm{~mm}$ diameter was impinged on distilled water, the deeply penetrating behavior of a columnar bubble ahead of the particle was also observed. The particle was separated from the columnar bubble at some position in liquid and slowly settled down at its terminal velocity, while the bubble quickly floated up after the maximum penetration.

\section{Discussion}

\section{Hypothetical Maximum Penetration Length}

If a force balance is applied to a particle moving down in a liquid, the following equation is derived.

$$
\begin{gathered}
-4 / 3 \pi R_{p}^{3} \rho_{p}\left(d V_{l} / d t\right)=-4 / 3 \pi R_{p}^{3} \rho_{p} g+4 / 3 \pi R_{p}^{3} \rho_{l} g \\
+\pi R_{p}^{2 *} 1 / 2 \rho_{l} V_{l}^{2} \cdot C_{D}+1 / 2 \cdot 4 / 3 \pi R_{p}^{3} \rho_{l}\left(d V_{l} / d t\right)
\end{gathered}
$$

where, the left hand side: the rate of change of momentum of a particle

$V_{l}$ : the particle velocity in the liquid

$R_{p}$ : the radius of the particle.

In the right hand side, the first to fourth terms are the force of gravity, buoyancy force, drag force and fluid inertia force (virtual mass term), respectively.

If the kinetic energy of a particle is not dissipated at the impact on the liquid surface, the particle would penetrate into the liquid with retaining velocity at the liquid surface just before penetration.

The penetration depth under this condition would be maximum. The hypothetical maximum penetration depth $(L)$ of a particle can be calculated by double integration of Eq. (1) by putting $V_{l}=V_{p o}$ at $t=0$, and shown in Figs. 7 to 10 as dashed curves. The equation for the maximum penetration depth is;

$$
\begin{aligned}
L & =-\left[\left[4 d_{p}\left(\rho_{p}+1 / 2 \rho_{l}\right)\right] /\left(3 C_{D} \cdot \rho_{l}\right)\right] \\
& * \ln \left[\cos \left(\tan ^{-1} \sqrt{\left(3 C_{D} \rho_{l}\right) /\left[4 d_{p}\left(\rho_{l}-\rho_{p}\right) g\right]} V_{p o}\right)\right]
\end{aligned}
$$

where, $C_{D}=0.44 .^{9)}$

Though $L$ increases with increasing $V_{p o}$, the rate of increase of $L$ gradually decreases with increasing $V_{p o}$.

\section{Particle Penetration Behavior}

\section{Mathematical Model}

Table 3 represents a typical example of dependence of the kinetic energy on the impact velocity. In the larger range of $V_{p o}, U_{\text {in }}$ is different from $U_{\text {wet }}$ about 5 orders of magnitude.

When particle impacts on a liquid surface, spitting, cavity and cylindrical wave formation, as shown in Fig. 11(a), take place. In addition to them, heat generation should be also taken into account. A part or most of kinetic energy of the impinging particle is consumed by these phenomena at the surface. After the impact by the particle, wetting of the impinging particle occurs and the particle penetrates into the liquid and energy consumption in the bulk of the liquid follows.

A mathematical model of energy consumption by the phenomena at the surface and motion in the bulk of liquid is described in the following sections.

\section{Gavity and Gylindrical Wave Formation}

If the particle velocity was less than a critical velocity, only a cavity formed without accompanying columnar bubble penetration. Only the particle deeply penetrated into the liquid. The mathematical 
Table 3. The comparison of the observed and calculated values.

\begin{tabular}{|c|c|c|c|c|}
\hline & $\begin{array}{c}\text { Impact } \\
\text { velocity } \\
V_{p o} \\
(\mathrm{~m} / \mathrm{s})\end{array}$ & $\begin{array}{c}\text { Cavity } \\
\text { radius } \\
R \\
(\mathrm{~mm})\end{array}$ & $\begin{array}{c}\text { Kinetic } \\
\text { energy } \\
\text { at impact } \\
U_{\text {in }} \\
\left(10^{-5} \mathrm{~J}\right)\end{array}$ & $\begin{array}{l}\text { Energy re- } \\
\text { quired for } \\
\text { wetting* } \\
U_{\text {wet }} \\
\left(10^{-7} \mathrm{~J}\right)\end{array}$ \\
\hline Sample A & 3.4 & 2.8 & 8.3 & 1.0 \\
\hline Sample B & 42.2 & 4.5 & 1228.3 & 1.0 \\
\hline
\end{tabular}

* Contact angle of the polystyrene : $87^{\circ}$

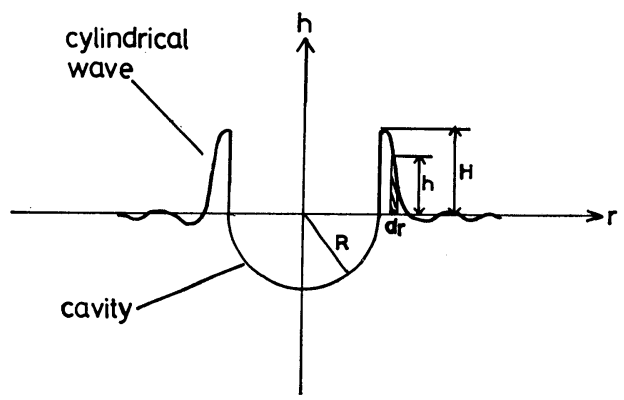

(a) Mathematical model
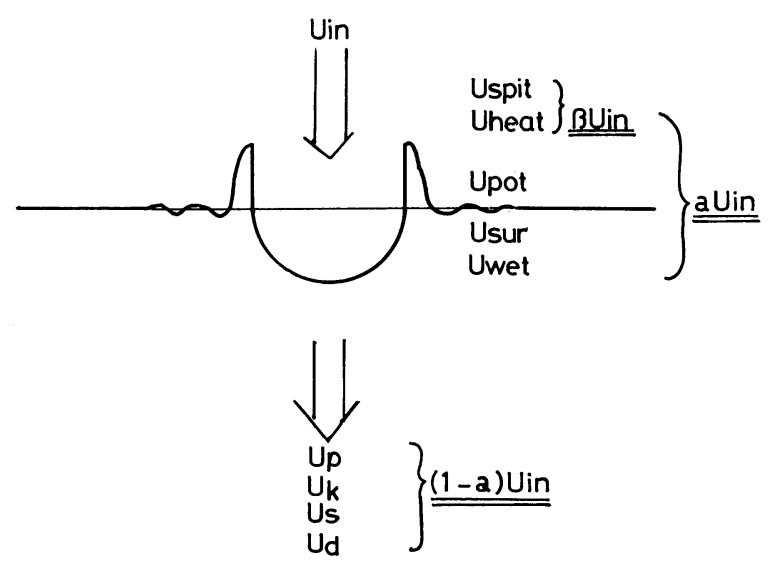

(b) Energy balance

Fig. 11. Schematic drawing of the penetration behavior for mathematical model

model to predict energy consumption for this process was obtained from consideration of the energy balance as shown in Fig. 11(b) and under the following assumptions :

(1) The transfer of energy from the particle to the target liquid occurs instantaneously.

(2) The surface energy for wetting the impinging particle is small and may be neglected.

(3) The shape of the cavity is hemispherical.

(4) The shape of the columnar bubble is cylindrical.

(5) The height of the cylindrical wave $(H)$ is $0.8 R$ (from the experimental results).

Energy equations for each energy term represented in Fig. 11(b) are established according to the model shown by Fig. 11 (a) as follows.

(I) Gravitational Potential Energy: $U_{\text {pot }}$

(1) Gavity formation: $U_{1}$

$$
U_{1}=1 / 2 \int_{0}^{R} 2 \pi r \cdot h d r \rho_{l} g h=(\pi / 4) g \rho_{l} R^{4}
$$

(2) Gylindrical wave formation: $U_{2}$

$$
U_{2}=1 / 2 \int_{R}^{\infty} 2 \pi r \cdot h d r \cdot \rho_{l} g h=0.11 \pi \rho_{l} g R^{4}
$$

where, $R$ : the radius of the cavity.

The height of a cylindrical wave $(h)$ is a function of the distance $(r)$ and assumed to be given by

$$
h=C_{1} / r_{1}^{3}+C_{2} / r_{2}^{4} .
$$

The first term was set to satisfy the condition (i). The second term is the higher order term to represent the damping shape of the cylindrical wave.

(i) $\int_{R}^{\infty} 2 \pi r \cdot h d r=2 / 3 \pi R^{3}$

B.C.

(ii) At $r \rightarrow \infty: h=0$

(iii) At $r=R: h=0.8 R$

where the height of the cylindrical wave must be determined from three conditions.

(i) The volume of the cylindrical wave is equal to that of the cavity.

(ii) The height of the cylindrical wave is zero at infinity.

(iii) The height of the cylindrical wave at $r=$ $R(H)$ is determined by experiment.

$$
h=-2 / 15 R^{4} / r^{3}+14 / 15 R^{5} / r^{4}
$$

Thus, $U_{\text {pot }}$ is given by $U_{\text {pot }}=U_{1}+U_{2}$.

(II) Surface Energy due to Newly Generated Surface: $U_{\text {sur }}$

(a) Net generated surface in cylindrical wave

(1) Surface area of cylindrical wave: $S_{1}$

$$
S_{1}=2 \pi \int_{R}^{\infty} r\left[1+(d h / d r)^{2}\right]^{1 / 2} d r-2 \pi \int_{R}^{\infty} r d r=0.8 \pi R^{2}
$$

(2) The area of the vertical wall of cylindrical wave: $S_{2}$

$$
S_{2}=2 \pi R \cdot H=1.6 \pi R^{2}
$$

(b) Net generated area of the cavity: $S_{3}$

$$
S_{3}=2 \pi R^{2}-\pi R^{2}=\pi R^{2}
$$

Thus, $U_{\text {sur }}$ is given by $U_{\text {sur }}=\left(S_{1}+S_{2}+S_{3}\right) \cdot \sigma$, where $\sigma$ is the surface tension. $U_{\text {pot }}$ and $U_{\text {sur }}$ were calculated by using $R$ which was obtained by analysis of high speed video films.

The result of calculation reveals that $U_{\text {pot }}$ is negligibly small in comparison with the other dissipation energy terms over all range of $V_{p o}$ and $U_{\text {sur }}$ takes $7 \sim$ $9 \%$ of the initial kinetic energy of the particle $\left(U_{\text {in }}\right)$ in a smaller range of $V_{p o}$. In a larger range of $V_{p o}$, both $\left(U_{e}=U_{\text {pot }}+U_{\text {sur }}\right)$ contributions become negligible. Figure 14 represents these results.

\section{Penetration of Golumnar Bubbles}

As shown in Fig. 2, a columnar bubble approximately 1.5 times the particle diameter penetrated more deeply into the liquid than the particle with im- 
pact velocities greater than the critical.

It has been indicated that the system with a Weber number larger than the critical number, which varies between 2 and 4 depending upon the characteristics of the system, becomes unstable. ${ }^{10,11)}$ In the present case, the Weber number is always larger than 2.8 , so the interface of the columnar bubble was unstable, and after some time interval the closing by oscillation occurred. Thus, the columnar bubble became disconnected with the free surface but still kept moving into the liquid.

The following fact can be obtained by considering the previous statement in Fig. 4;

(1) The deeply penetrating columnar bubble is surrounded by the ink from the free surface.

(2) The layer of ink simultaneously moves into the liquid in company with penetration of the columnar bubble.

It is, therefore, inferred that the motion of the ink layer around a columnar bubble reflects the deformation and simultaneous motion of a portion of the liquid surface by impingement of a particle, which resulted in the entrainment of the gas and formation of narrow pipe (i.e., columnar bubble).

The columner bubble is accompanied with a thin liquid boundary moving into the liquid.

Figure 12 shows a schematic representation of the above mentioned process. On the basis of the idea of Fig. 12, a mathematical model to predict the energy required for moving a boundary layer of liquid to the maximum penetration length of the columnar bubble is presented as follows.

The assumptions are:

(1) The columnar bubble and the boundary layer of the liquid surrounding the columnar bubble move together until the maximum penetration is achieved.

(2) The standing bulk liquid is regarded as a rigid wall.

(3) At the cylindrical rigid wall, the surface is approximately regarded as a flat plate with a width of b. ${ }^{12)}$

According to the boundary layer flow theory the accepted formulas for flat-plate flow $\operatorname{are}^{13)}$ :

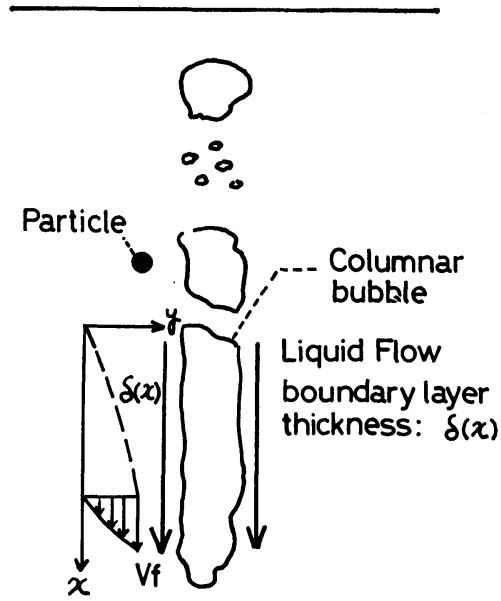

Fig. 12. Schematic representation of liquid flow with columnar bubble. $\delta / x \approx 5.0 R e_{x}^{-1 / 2} \ldots .$. for laminar flow

$\delta / x \approx 0.16 R e_{x}^{-1 / 7} \ldots . .$. for turbulent flow

The wall shear stress was calculated by Blasius. ${ }^{14)}$

$$
\tau_{w}(x)=0.332 \rho_{l}^{1 / 2} \mu^{1 / 2} V_{f}^{1.5} x^{-1 / 2},
$$

where, $V_{f}$ : the average flow velocity of the liquid.

The total drag force for liquid flow equals to the integrated wall shear along the plate

$$
D(x)=b \int_{0}^{x} \tau_{w}(x) d x=0.664 b \rho_{l}^{1 / 2} \mu^{1 / 2} V_{f}^{1.5} x^{1 / 2}
$$

The total dissipation energy for liquid flow is calculated as follows:

$$
U_{d}=\int_{0}^{L a} D(x) d x,
$$

where, $L a$ : the penetration length of the columnar bubble.

The order of magnitude of boundary layer thickness $(\delta)$ calculated by using Eq. (10) was approximately $1 \mathrm{~mm}$. Thus, the dissipation energy caused by liquid flow was less than $10 \%$ of the kinetic energy of the particle at impact.

\section{Calculation of the Energy Transfer Coefficient}

Figure 13 shows the relation between the particle impact velocity $\left(V_{p o}\right)$ and the initial velocity of the particle in the liquid $\left(V_{l o}\right)$, which was obtained by solving Eq. (1) with known values of $L$ obtained from video films.

The energy transfer coefficient $(\alpha)$ is defined as the ratio of the consumed energy on impingement to the kinetic energy of the particle at the liquid surface just before impingement, that is the energy fraction solely consumed at the surface and not brought into the bulk of the liquid.

Definition: $\alpha=\left[U_{\text {in }}-\left(U_{k}+U_{p}+U_{s}+U_{d}\right)\right] / U_{\text {in }}$

where, $U_{\text {in }}:$ kinetic energy of the particle at impact $U_{k}$ : kinetic energy of the particle in the liquid

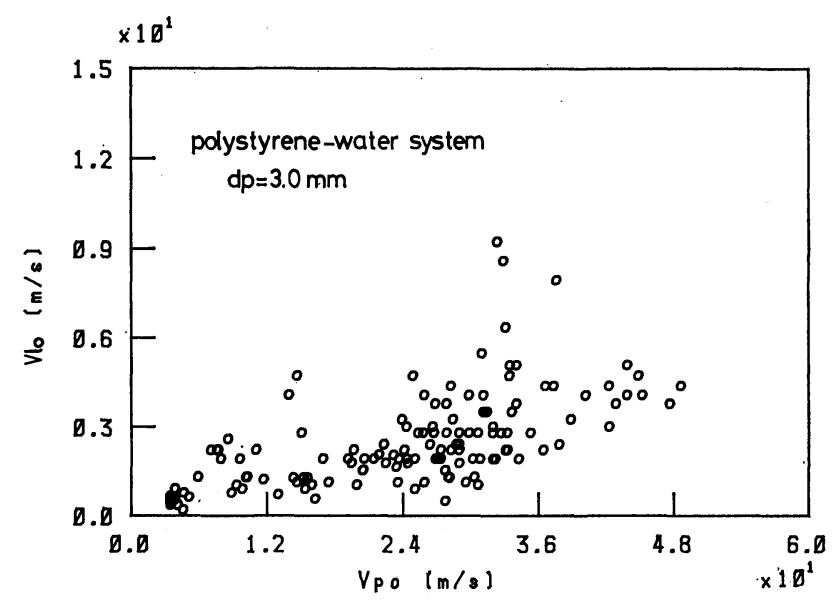

Fig. 13. Relation between the particle velocity in liquid $\left(V_{l o}\right)$ and impact velocity $\left(V_{p o}\right)$. 
$U_{p}$ : potential energy of the columnar bubble

$U_{s}$ : surface energy of the columnar bubble

$U_{d}$ : total dissipation energy for liquid flow.

The value of $U_{k}$ was determined by using $V_{l o}$. The values of $U_{p}$ and $U_{s}$ were obtained by the maximum penetration depth and the volume of the columnar bubble. Figure 14 represents the dependence of fractional contribution of each energy term on impact velocity. Contribution by $U_{k}$ is quite small especially in the higher region of $V_{p o}$.

The dependence of $\alpha$ on $V_{p o}$ is shown in Fig. 15. Although the value of $\alpha$ scattered in the region of smaller $V_{p o}$, it is approximately 0.7 and increases up to 0.95 with increase in $V_{p o}$. This result suggests that most of $U_{\text {in }}$ is consumed at the surface and that only a small portion of kinetic energy can be brought into the liquid especially when the impact velocity of the particle is quite large.

\section{Fraction of Undetermined Dissipation Energy}

The fraction of undetermined dissipation energy $(\beta)$ is defined as the ratio of the sum of the spitting and heat generation, which are not calculable, to the kinetic energy of the particle at the liquid surface and calculated by the following equation

Definition: $\beta=\left[\alpha U_{\text {in }}-\left(U_{\text {pot }}+U_{\text {sur }}+U_{\text {wet }}\right)\right] / U_{\text {in }}$,

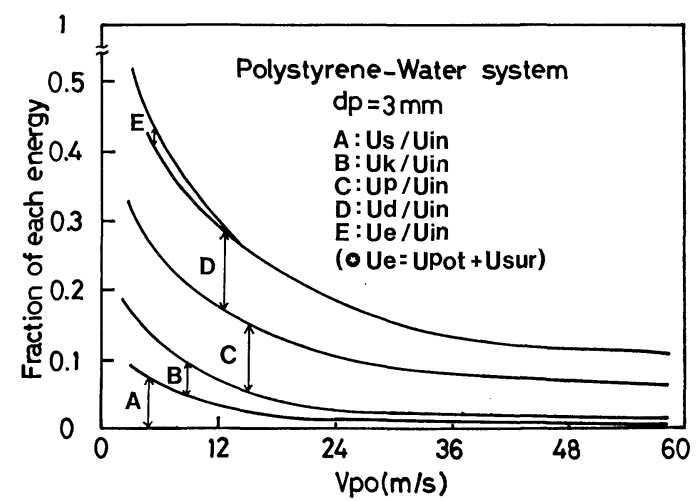

Fig. 14. Dependence of contribution of each dissipation mechanism to initial kinetic energy on impact velocity $\left(V_{p o}\right)$.

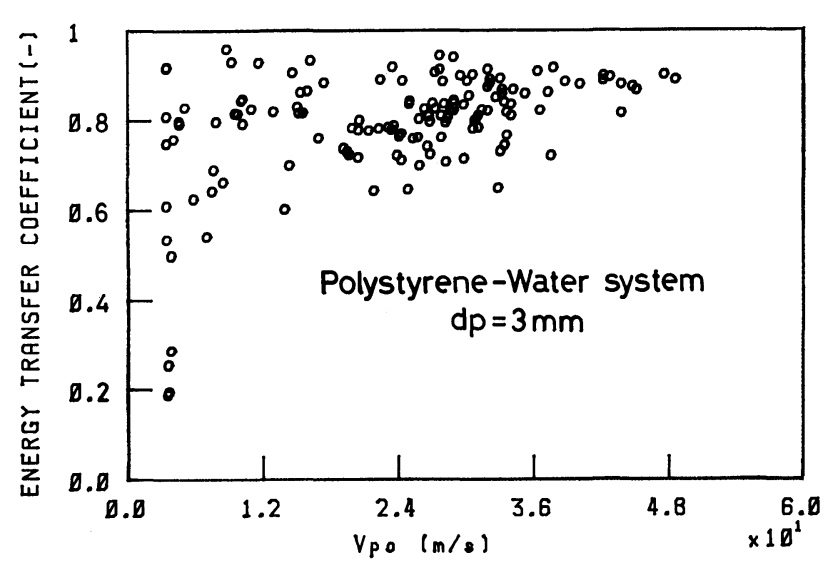

Fig. 15. Variation of the energy transfer coefficient with impact velocity $\left(V_{p o}\right)$. where, $\alpha$ : energy transfer coefficient

$U_{\text {wet }}$ : wetting energy.

Figure 16 shows the dependency of $\beta$ on the particle impact velocity. The value of $\beta$ is in scatter in the smaller region of $V_{p o}$. However, in the larger region of $V_{p o}, \beta$ is almost equal to $\alpha$ and thus the undetermined part of energy becomes important. $U_{\text {in }}$ of $\mathrm{B}$ in Table 3 corresponds to the thermal energy to heat up the same volume of water by $0.2^{\circ} \mathrm{C}$.

In conclusion, when a particle impinges on liquid surface with a large impact velocity, most of kinetic energy of the particle is consumed by the surface work, probably as spitting and heat generation, at the liquid surface.

\section{Penetration Behavior in Powder Injection}

As discussed above, most of the kinetic energy of an impacting particle is dissipated at its point of impingement on the liquid surface and only a few percent of kinetic energy is left for the particle to penetrate further into the liquid. This could be also the case for the molten steel system, since the similarity in term of Reynolds number would strictly hold in the region of very high impact velocity. Thus it can be concluded from the present study that the higher particle impact velocity can not be an effective means to make a particle penetrate deeper into the liquid including molten steel.

However, it has been observed sometimes in industry that the penetration length of a particle seems to be large enough even to erode the refractory of the bottom of converter or runner. ${ }^{15)}$

The difference between the present study and these practical processes may be attributed to [1] single particle $v s$. mass of powder and [2] with or without accompanying gas jet.

As shown in Fig. 6, the amount of the entrained gas was $10 \sim 70$ times larger than the particle volume.

In the case of powder injection, therefore, it is anticipated that a large amount of gas becomes entrained in the liquid, which may result in a lower density of the liquid and easier penetration of particles.

An experiment was conducted to clarify the effect

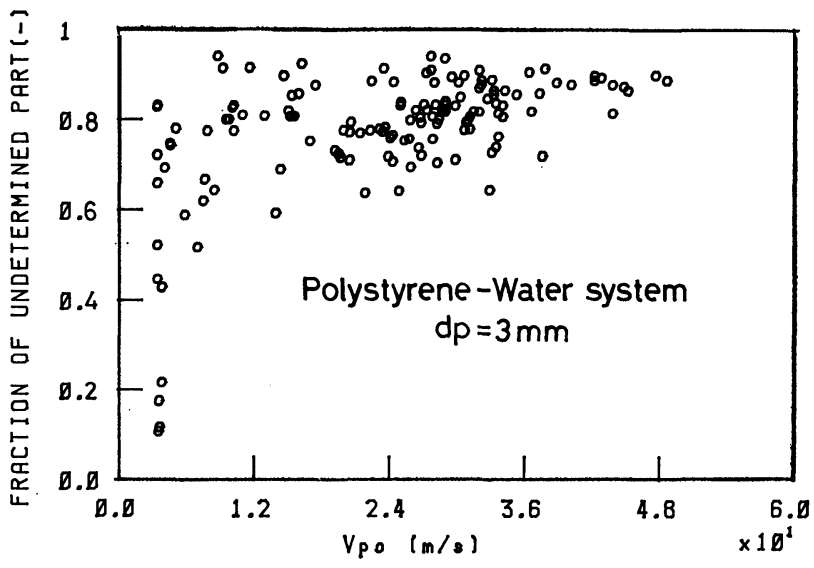

Fig. 16. Variation of the fraction of undetermined dissipation energy terms with impact velocity $\left(V_{p o}\right)$. 
of gas holdup in the liquid on the penetration length of a single particle. The experimental set-up and procedure were explained in Chap. II. The gas holdup increased by increasing electric current $(50,100$, $150,200 \mathrm{~mA}$ ), as shown in Table 2.

The dependence of penetration length on gas holdup is shown in Fig. 17. Here, about $0.065 \%$ of the gas volume corresponding to $0.065 \%$ reduction of density was attained by applying $200 \mathrm{~mA}$. For comparison, the average penetration depths of bubbles and particles in Fig. 7 are represented by triangles.

It is evident that the penetration length of particles becomes larger and that of bubbles becomes less if the liquid has an appreciable gas holdup. It is not clear whether the deeper penetration is attributed to the reduction of apparent liquid density which may reduce the resistance to the particle movement or to the introduction of gas holdup itself which may reduce the energy consumption at the surface. However it may be suggested from this simple experiment that the introduction of bubbles in liquid with increasing gas holdup provide an effective way to obtain deep penetration of powders into liquids.

In practical powder injection processes, moreover, the gas jet itself can contribute to a large gas holdup by simultaneous entrainment of gas in the liquid, which would guarantee considerable penetration of powders into the liquid.

In addition, it has been found that the motion of the liquid itself can carry the particles deep into the liquid and is regarded to be very important also from a practical point of view.

Consequently, it was inferred that in the powder injection processes, the penetrating condition of the particle becomes very favorable because of building up of gas-liquid two phase region in the liquid phase.

\section{Conclusions}

(1) Penetration behavior of a particle impinged on a liquid surface was observed with a high speed video and camera.

There was a critical velocity at impact beyond which gas is entrained as a columnar bubble.

When the impact velocity is small, a cavity is formed upon impact and only the particle penetrates into the liquid through the cavity surface. In this regime, the energy required for cavity formation $\left(U_{e}=\right.$ $\left.U_{\text {pot }}+U_{\text {sur }}\right)$ was $7 \sim 9 \%$ of the kinetic energy at impact of the liquid surface $\left(U_{\mathrm{in}}\right)$.

(2) In the case of particle impact velocities greater than the critical, the particle separates from the bubble at some depth and the columnar bubble penetrates more deeply into the liquid than the particle in both the distilled water-polystyrene and glycerinpolystyrene system.

The residence time of the particle in the distilled water-polystyrene system was approximately on average $2.3 \mathrm{~s}$ which was 7 times longer than the bubbles.

(3) Particle penetration depth increases with increasing impact velocity, but the effect decreases with increasing velocity.

(4) When a particle impacts on liquid surface

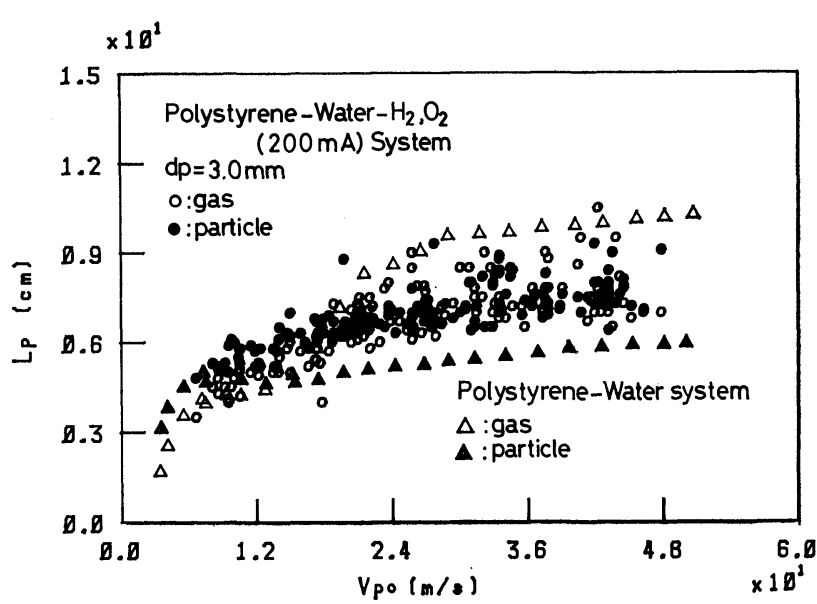

Fig. 17. Change of the penetration lengths of gas and particle with impact velocity, showing the effect of gas holdup.

with a large velocity, most of the kinetic energy is consumed by dissipation work, mainly spitting and heat generation, and only a small fraction is brought into the liquid bulk with the particle.

It is concluded that the higher particle velocity is not good means to increase the penetration depth.

(5) In particle impinging, the amount of the entrained gas is $10 \sim 70$ times of the particle volume.

It was found that a slight decrease in density of the liquid by increasing gas holdup favored the deeper penetration of particles into the liquid.

Reduction of apparent liquid density by increasing gas holdup or even introduction of gas holdup in the liquid with entrained gas or gas jet seems to be the most effective way to obtain deep penetration of powders into liquids.

\section{Acknowledgements}

The authors are very much obliged to Dr. Kimihisa Ito, Research Inst. of Mineral Dressing and Metallurgy, Tohoku Univ., for his most valuable contribution in setting up the experimental apparatus and the initial part of the present study. They are grateful to Prof. S. Kobayashi and Dr. M. Ishigaki of the Institute for their help in carrying out the experiment and discussion. Prof. H. Hashimoto, Inst. of High Speed Mechanics, Tohoku Univ., for his valuable suggestions and Prof. D. W. Davenport of Univ. of Arizona for critical reading of the manuscript as well as Fukuyama Research Laboratory, Steel Research Center, Nippon Kokan K.K. for permission of use of their high speed camera are also acknowledged.

\section{REFERENGES}

1) T. A. Engh, K. Larsen and K. Venas: Ironmaking Steelmaking, 6 (1979), 268.

2) L. R. Farias and G. A. Irons: Metall. Trans. B, 16B (1985), 211.

3) T. A. Engh, H. Sandberg, A. Hultkvist and L. G. Norberg: Scand. J. Metall., 1 (1972), 108.

4) Y. Ozawa and K. Mori: Tetsu-to-Hagané, 69 (1983), 753.

5) T. A. Engh and H. Bertheussen: Scand. J. Metall., 4 (1975), 241.

6) E. Kimura: Trans. Iron Steel Inst. Jpn., 23 (1983), 522. 
7) G. A. Irons and B. H. Tu: Proceedings of Scaninject III Conference Luleå, June 15-17, 1983, MEFOS and Jernkontoret, Sweden, (1983), 11.1-11.29.

8) L. R. Farias and D.G.G. Robertson: Proceedings of 3rd Process Technology Conference, Pittsburgh, ISS-AIME, (1982), 206-220.

9) J. Szekely: Fluid Flow Phenomena in Metal Processing, Academic Press, New York, (1979), 256-257.

10) S. Hu and R. C. Kintner: AIChE J., 1 (1955), 42.
11) E. Goester: Z. Angew. Phys., 13 (1961), 252.

12) L. D. Landau and E. M. Lifshitz: Fluid Mechanics, Pergamon Press, London, (1963), 163.

13) F. M. White: Fluid Mechanics, McGraw-Hill, New York, (1979), 400.

14) F. M. White: Fluid Mechanics, McGraw-Hill, New York, (1979), 410.

15) K. Yamada, K. Iwasaki, H. Ito, G. Nakatani and M. Ohtsuki: Tetsu-to-Hagané, 71 (1985), 1615. 